\title{
Outcome of Guillain-Barr'e Syndrome after Five Sessions of Plasmapheresis
}

\author{
Salma Haji
}

\begin{abstract}
OBJECTIVE: To find out the outcome of Guillain-Barr'e Syndrome after five sessions of Plasmapheresis. METHODOLOGY: This was prospective, descriptive observational study conducted from January 2018 July 2019 in neurology ward 28, JPMC Karachi. Patients above 12 years of age diagnosed as Guillain-Barr'e Syndrome admitted through OPD and emergency were included. Patients diagnosed on the basis of clinical findings, cerebrospinal fluid detail report and nerve conduction studies. In Cerebrospinal fluid detail report, albuminocytologic dissociation was observed. Double lumen catheter was passed and plasmapheresis was done on every alternate day, 1.5-2.0 liters of plasma was removed and replaced by 4 FFPS, $1000 \mathrm{ml}$ of normal saline and $500 \mathrm{ml}$ of haemocoel for patients of diabetic neuropathy. Outcome of the patients was recorded as complete recovery, residual deficit and mortality. Patients were followed for six months. Data was analyzed by SPSS version 24.

RESULTS: Eighty nine patients of Guillain-Barr'e Syndrome were enrolled. Male patients were 60 $(67.42 \%)$ whereas female patients were $29(32.58 \%)$. There were 15 patients $(16.85 \%)$ within $12-20$ years of age, 46 patients $(51.68 \%)$ in $21-40$ years of age, 19 patients $(21.34 \%)$ from $41-60$ years of age, 9 patients $(10.11 \%)$ were above 60 years of age, 5 patients $(5.62 \%)$ died while $64(71.91 \%)$ patients were fully recovered after plasma exchange. Twenty patients $(22.47 \%)$ were partially recovered with residual motor weakness.
\end{abstract}

CONCLUSION: Plasma exchange is a good treatment modality for the Guillain-Barr'e Syndrome patients.

KEYWORDS: Guillain-Barr'e Syndrome, Plasmapheresis, Neuropathy.

This article may be cited as: Haji S. Outcome of Guillain-Barr'e Syndrome after Five Sessions of Plasmapheresis. J Liaquat Uni Med Health Sci. 2019;18(04):281-4. doi: 10.22442/jlumhs.191840643

\section{INTRODUCTION}

Guillain-Barr'e Syndrome (GBS) an autoimmune disease is a common cause of generalized progressive paralysis. Guillain-Barr'e Syndrome is actually a poly radiculoneuropathy with available clinical course. Guillain-Barr'e Syndrome is mainly a post infectious disease manifested by rapid onset progressive, symmetrical weakness of the extremities. About $25 \%$ of Guillain-Barr'e Syndrome patients may develop respiratory failure and sometimes autonomic dysfunction ${ }^{1}$.

Diagnosis is usually made clinically and once cerebrospinal fluid detail report, albuminocytologic dissociation and electrophysiological studies are done to make diagnosis ${ }^{2}$. Plasma exchange in Guillain-Barr'e Syndrome shortens the duration and severity of illness. Plasma exchange is a procedure in which patients' blood is passed through machine, which separates the different type soft blood cells and returns all the cellular components like RBC, WBC and platelets along with either donor plasma or a replacement solution. This procedure removes substances which attacks myelin. Plasma exchange is very beneficial when started within a week after illness but it was also beneficial in patients treated up to 30 days after disease onset ${ }^{3}$.

Plasma exchange is a better option for patients of Guillain-Barr'e syndrome because of negligible complications and highly cost effective ${ }^{3}$. It reduces the economic burden as compared to Intravenous globulin. Guillain-Barr'e Syndrome has no cure but plasma exchange can reduce the duration of illness and aids patient's recovery ${ }^{4}$. Spontaneous recovery may occur, however up to $75 \%$ of patients may develop sequelae ${ }^{4}$. Mortality in Guillain-Barr'e Syndrome patients is $5 \%$. Plasma exchange removes antibodies from the blood stream. In Guillain-Barr'e Syndrome 5-6 therapeutic plasma exchange over 14 days are recommended with $5 \%$ albumin replacement. Patients of Guillain-Barr'e Syndrome benefit from plasmapheresis irrespective of initial severity so it should be started early as more benefit will occur. Plasma exchange reduces the risk of developing respiratory failure.

The rationale of study is that plasmapheresis treatment of Guillain-Barr'e Syndrome is available only in few centers and rarely the studies are done on this procedure in Pakistan. We are doing plasmapheresis 
in patients of Guillain-Barr'e Syndrome in J.P.M.C. Karachi to look for prognosis of Guillain-Barr'e Syndrome patients in our setup.

\section{METHODOLOGY}

This prospective, observational study was conducted from January 2018 till July 2019 at Jinnah post Graduate Medical Centre Karachi Ward-28. Eighty nine patients who were above 12 years of age both male and female were admitted emergency and OPD were diagnosed as Guillain-Barr'e Syndrome on clinical findings and lab investigations like Cerebrospinal fluid D/R and nerve conduction studies were included. Complete history and physical examination was done. Patients presented with quadriparesis, difficulty in breathing, paraparesis and autonomic dysfunction were also included. Their investigations like lumber puncture were done. Albuminocytologic dissociation was observed in Cerebrospinal fluid D/R. Nerve Conduction Studies of all patients was done as well, before procedure of Blood Pressure, Pulse, Respiratory rate and temperature was recorded. Patients' consents were taken after explaining them the risks and benefits of the procedure. Double lumen catheter was passed in femoral vein or internal jugular vein. Plasma exchange was done in all patients. 4FFPs, in combination with normal saline 1 litre and haemocoel was given as a replacement fluid. 5 cycles of plasma exchange was done on alternate days and were completed. Post plasmapheresis, patient outcome was recorded as residual deficit or partial recovery and full recovery or mortality. After 2 weeks patients were discharged and were followed for 6 months in OPD to see the improvement in motor weakness. Results were analyzed on SPSS version 24 in frequency.

\section{RESULTS}

A total of 89 patients of Guillain-Barr'e Syndrome were enrolled in the study. Male patients were 60(67.42\%) whereas female patients were $29(32.58 \%)$, so male patients were predominantly involved. There were 15 patients $(16.85 \%)$ within $12-20$ years of age range. 46 patients $(51.68 \%)$ in $21-40$ years of age range, 19 patients $(21.34 \%)$ from $41-60$ years of age range, 9 patients $(10.11 \%)$ were above 60 years of age. Eighty four patients $(94.38 \%)$ were recovered after 5 cycles of plasma exchange and five patients $(5.62 \%)$ died due to respiratory failure and autonomic dysfunction. Fully recovered patients had Grade-5 power in all four limits while in partially recovered patients power improved from Grade-1 to Grade-3. In GBS, outcome of plasmapheresis is quick Figure I.
FIGURE I: NUMBER OF GUILLAIN-BARR'E SYNDROME PATIENTS

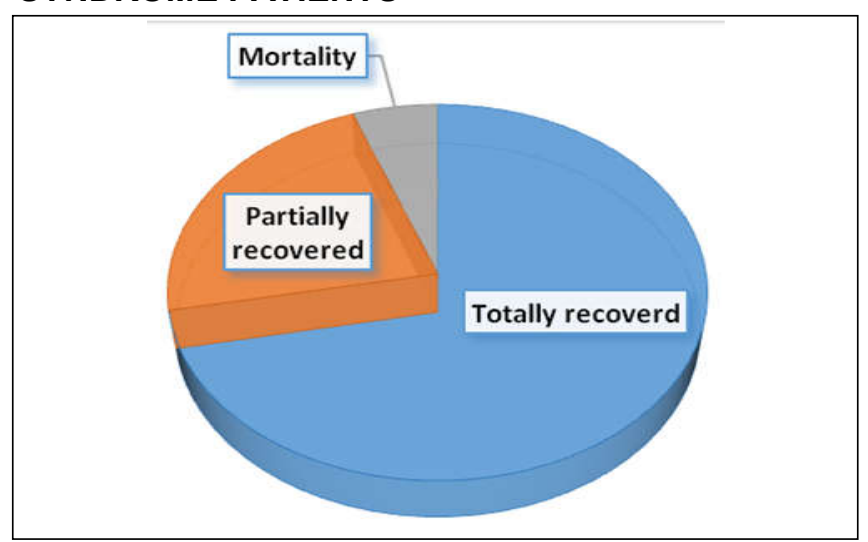

TABLE I: OUTCOME OF GBS PATIENTS AFTER FIVE CYCLES OF PLASMAPHERESIS

\begin{tabular}{|l|c|c|}
\hline \multicolumn{1}{|c|}{$\begin{array}{c}\text { Outcome of GBS } \\
\text { patients }\end{array}$} & $\begin{array}{c}\text { No of } \\
\text { Patients }\end{array}$ & Percentage \\
\hline Totally recovered & 64 & $71.91 \%$ \\
\hline Partially recovered & 20 & $22.47 \%$ \\
\hline Mortality & 5 & $5.62 \%$ \\
\hline Total no of patients & 89 & $100 \%$ \\
\hline
\end{tabular}

\section{DISSCUSSION}

Guillain-Barr'e Syndrome is a serious disorder in which peripheral nerves become inflamed which leads to paralysis and sensory symptom. Many people who develop Guillain-Barr'e Syndrome have a history of preceding viral illness like upper respiratory tract infections and intestinal infections campylobacter jejuni-antibodies against the infection target the nerves myelin sheath and causes Guillain-Barr'e Syndrome. Plasma exchange removes soluble factors including anti bodies from the blood and it is the treatment for patients of Guillain-Barr'e syndrome. Plasma exchange replaces the patients' plasma with an artificial plasma substitute usually on albumin solution ${ }^{6}$.

Cause of Guillain-Barr'e Syndrome is not known yet. Men are 1.5 times more affected than women ${ }^{6}$. In this study males were affected more than females. Campylobacter jejuni gastroenteritis may be the preceding illness responsible for Guillain-Barr'e Syndrome, ${ }^{7}$ therefore immune response can lead to cross infection on host's nerves. $70 \%$ patients have been reported to have this infection ${ }^{7}$.

After the acute phase of disease Guillain-Barr'e patients do well. Most patients achieve independent ambulation after 6 months $^{8}$. Mortality during acute phase of disease is less than $5 \%, 20 \%$ patients may 
have significant disability. Early identification of poor prognostic factors can lead to further treatment like plasma exchange. Early diagnostic findings in Guillain -Barr'e Syndrome include absent or prolonged $\mathrm{H}$ reflexes or frequency wave latencies ${ }^{10}$. The sural sparing pattern is considered specific for GuillainBarr'e Syndrome as compared to other polyneuropathies ${ }^{11}$. This pattern showed on intact sural sensory response with abnormal upper extremely sensory responses. Other findings depend on variants of Guillain-Barr'e Syndrome. Acute inflammatory demyelinating polyneuropathy more likely to have partial motor conduction block, temporal dispersion, slow conduction velocities, prolonged /absent F-wave latencies and prolonged distal latencies.

Autonomic Dysfunction is the main cause of morbidity and mortality attributable to Guillain-Barr'e Syndrome an involvement of respiratory muscles can lead to respiratory failures and death. In this study, mostly the cause of death was autonomic failure, like hypotension, hypertension, cardiac arrhythmia, and tachycardia.

Intravenous immunoglobulin's and plasma exchange are proven good treatment for Guillain-Barr'e Syndrome, but in spite of these treatments, the prognosis of Guillain-Barr'e syndrome is still not satisfactory. The zipper method of treatment seems to reduce the mortality. In this method plasma exchange is done by removing the plasma and replacement by albumin at the end of exchange. $4 \mathrm{gm} / \mathrm{kg} / \mathrm{Intravenous}$ immunoglobulin should be given. This method seems to reduce the mortality, speed up weaning from mechanical ventilation and shortens the hospital stay in severe Guillain-Barr'e Syndrome. But in this study we have not given the albumin as a replacement fluid and also intravenous immunoglobulin.

Spontaneous recovery in Guillain-Barr'e Syndrome may occur. About 5\% patients may develop ophthalmoplegia, ataxia and arreflexia. Autoimmune pathogenesis is strongly suggested by the presence of antibodies against the four gangliosides-GM, GDA, GT1A and Gqlb which differ by number and position of siolic acid. The observation of preceding infection with campylobacter jejuni suggests cross reactive antibodies may be a component in disease pathogenesis. There are several scales to evaluate the severity and prognosis of Guillain-Barr'e syndrome disability. Medical research council sum score (MRCS. Score) ARASMUS score ${ }^{3}$. GBS Respiratory insufficiency score and ARASMUS GBS outcome score. Most of the treatment goal is to lessen the severity of Guillain-Barr'e Syndrome illness and to assess the patients' recovery.

High quality ICU care remains the most important aspect of management of Guillain-Barr'e syndrome.
Plasma exchange and high dose Intravenous globulin are equally effective modes of treatment ${ }^{12}$. Physical therapy is advised to all patients when they are in the hospital. The mechanism, which intravenous immunoglobulin works in Guillain-Barr'e Syndrome is unclear. Intravenous globulin has minimal side effects like headache, skin reactions at infusion sites, flu-like symptoms, and aseptic meningitis, thromboembolic events such as pulmonary embolism due to increased viscosity of the blood are usually seen rarely ${ }^{13}$. The small volume plasma exchange has safety and feasibility with little side effects like sepsis, DVT and hypersensitivity reactions. Patients can be treated safely as compared to standard plasma exchange ${ }^{14}$. In this study we have not encountered any complications like pulmonary embolism and deep venous thrombosis.

Some people give the steroids alone in Guillain-Barr'e syndrome, but steroids are ineffective and can worsen the Guillain-Barr'e Syndrome, but steroids if given with Intravenous globulin has good effect on Guillain-Barr'e syndrome. Patients with Miller Fischer Variant also benefit from Intravenous globulin and $20 \%$ still unable to walk after $1 / 2$ year.

Many patients have pain, fatigue or other residual complaints which persists for months for sometimes years. Prognostic model for Guillain-Barr'e Syndrome are now available. The ARASMUS GBS respiratory scale predicts the chance for artificial ventilation. Modified ARASMUS GBS outcome scale predicts the chance to be able to walk after 6 months.

Guillain-Barr'e Syndrome patients with poor prognosis potentially might benefit from more intensive management. A large increase in serum IgG levels after standard Intravenous globulin treatment $4 \mathrm{gm} / \mathrm{kg} /$ B.W for 5 consecutive days seems to improve the Guillain-Barr'e syndrome. The aid GBS trial investigates second Intravenous globulin course improves outcome in GBS patients with poor outcome. Patients with Guillain-Barr'e Syndrome especially need excellent multidisciplinary care to prevent fatal complications, this shows Guillain-Barr'e Syndrome need careful monitoring of cardiac and respiratory function. $25 \%$ of severely involved patients require mechanical ventilation and regularly evaluation of vital capacity and timely transfer to an ICU. They need early prophylaxis for DVT, cardiac and hemodynamic monitoring, Pain management, possible management of bladder dysfunction. Complications like choking and airway obstruction may lead to death in ICU patients ${ }^{15}$. Physiotherapy during early course of disease should be started. Rehabilitation should be encouraged in recovery phase. For severe fatigue, pain killers and physical training is very beneficial. We started early physiotherapy. 
The limitation in this study was that if we include the children below 12 years of age it can change the mortality and prognosis. Secondly, most patients presented earlier and plasmapheresis was done immediately so the results are better.

\section{CONCLUSION}

Plasmapheresis five cycles in Guillain-Barr'e Syndrome proved to be a good modality of treatment with good outcome of results.

Ethical permission: Jinnah Post Graduate Medical Centre Karachi, Letter No. 18153, Dated: 18-04-2019

Conflict of Interest: Authors of the study have no conflict of interest to declare.

Funding: There was no Grant or other financial support for this project.

\section{REFERENCES}

1. Christine verboon, Pieter A, Bart C Jcobs. Treatment dilemmas in Guillain Barr'e Syndrome. J Neurol Neurosurg Psychiatry. 2017; 88(4): 34652. doi: 10.1136/jnnp-2016-314862.

2. van den Berg $B$, Walgaard $C$, Dranthen J, Fokke C, Jacobs BC, van Doorn PA. Guillain-Barr'e Syndrome: pathogenesis, diagnosis, treatment and prognosis. Nat Rev Neurol. 2014; 10(8): 46982. doi: 10.1038/nrneurol.2014.121.

3. Mittal M, Agrawal J, Singh A. Therapeutic plasma exchange in Guillain Barr'e Syndrome, an experience of our centre. Int J Adv Med. 2016; 3(4): 829 -31. doi:10.18203/2349-3933.ijam20162883.

4. Iyer RR, Shah $\mathrm{PH}$, Suri SK. Reducing the Economic burden in Management of Guillain Barr'e Syndrome using modified plasmapheresis. Asian J Transfus Sci. 2016; 10(2): 118-21.

5. Prasad HB, Borse RT, Avate AN, Palasdeokar APIM. Prognostic Indicators of response to plasmapheresis inpatients of Guillain Barre Syndrome. J Assoc Physicians India. 2017; 65(4): 32-36.

6. Sudulagunta SR, Sodalagunta MB, Sepehrar M, Khorram H, Banaglore Raja SK, Kothandapani S, et al. Guillain- Barr'e Syndrome: clinical profile and management. Ger Med Sci. 2015; doc 13: Doc 16. . doi:10.3205/000220.

7. Nguyen TP, Taylor RS. Guillain Barre Syndrome. Stat Pearls, NCBI Bookshelf [Internet]. 2018.

8. Ortiz-Salas $P$, Velez Van Meerbeke $A$, Galvis-Gomez CA, Rodriguez Q JH. Human Immunoglobin versus Plasmapheresis in GuillainBarre Syndrome and Myasthenia Gravis. A MetaAanalysis . J Clin Neuromuscul Dis. 2016; 18(1): 1 -11. doi: 10.1097/ CND.0000000000000119.

9. Walgaard C, Lingsma HF, Ruts L, Drenthen J , van Koningsveld R, Garssen MJ, et al -Prediction of respiratory insufficiency in Guillain-Barr'e Syndrome. Ann Neurol. 2010; 67(6): 781-7. doi: 10.1002/ana.21976.

10. Susuki K, Nishimoto Y, Yamada M, Baba M, Ueda $\mathrm{S}$, Hirata $\mathrm{K}$, et al. Acute motor axonal neuropathy Rabbit Model: immune attack on nerve root axons. Ann Neurol. 2003; 54(3):383-8.

11. Kesici S, Tanyildiz M, Yetimakman F, Bayrakci B. A novel treatment strategy for severe Guillain-Barr'e Syndrome: Zipper method. J Child Neurol. 2019; 34(5): 277-83. doi:10.1177/ 0883073819826225

12. Schawartz J, Winters JL, Padmanabhan A, Balogun RA, Delaney M, LInenberger ML, et al. Guidelines on the use of Therapeutic Apheresis in Clinical Practice-Evidence Based approach from the writing committee of the American Society for Apheresis: The Sixth Special Issue. J Clin Apheresis. 2013; 28: 145-284.

13. Koc AF, Kilic NB, Yerdelen D, Bozemir $H$. GuillainBarre Syndrome; etiology, clinical findings and therapeutic plasma exchange. J Neurol Sci. 2005; 22: 267-73.

14. Islam B, Islam Z, Rahman S, Endtz HP, Vos MC, van der Jagt $M$, et al. Small volume plasma exchange for Guillain-Barre Syndrome in resource -limited settings: a phase II safety and feasibility study. BMJ Open. 2018; 8(8): e022862. doi.10.1136/bmjopen-2018-022862.

15. van Doorn PA. Current treatment in Guillain-Barr'e Syndrome and Myasthenia gravis. $4^{\text {th }}$ congress of the European Academy of Neurology, Lisbon-Portugal, June 16-19, 2018.

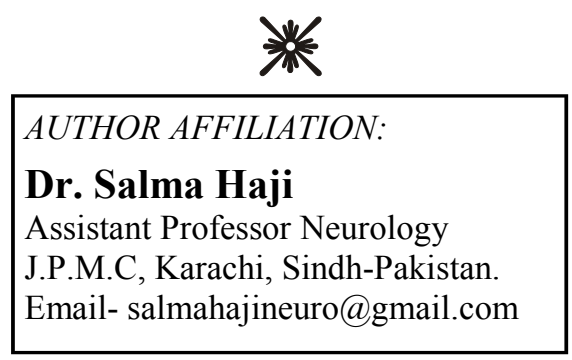

